\title{
Interpretive analogies between quantum and statistical mechanics
}

\section{D. $\operatorname{McCoy}^{1}$ (D)}

Received: 25 August 2018 / Accepted: 31 October 2019/Published online: 10 January 2020 (c) The Author(s) 2020

\begin{abstract}
The conspicuous similarities between interpretive strategies in classical statistical mechanics and in quantum mechanics may be grounded on their employment of common implementations of probability. The objective probabilities which represent the underlying stochasticity of these theories can be naturally associated with three of their common formal features: initial conditions, dynamics, and observables. Various well-known interpretations of the two theories line up with particular choices among these three ways of implementing probability. This perspective has significant application to debates on primitive ontology and to the quantum measurement problem.
\end{abstract}

Keywords Statistical mechanics - Quantum mechanics · Primitive ontology · Measurement problem

\section{Introduction}

A perusal of the literature reveals that rather little has been written down concerning the analogies between interpretations of quantum mechanics and of statistical mechanics - though such analogies have hardly escaped notice. Indeed, interpretive work on the two theories has proceeded largely independently. My aim in this paper is to go some way in rectifying the omission by drawing out these analogies explicitly, in particular by showing how they may be grounded on the way in which the theories' stochasticity, as I will call it, is represented and interpreted, with the further aims of showing how this integrative perspective can shed light on current issues in the philosophy of physics and suggest fruitful possibilities for future investigation.

Quantum mechanics and classical statistical mechanics are both theories of statistical physics, whose empirical content depends on the objective probabilities that

C. D. McCoy

casey.mccoy@philosophy.su.se

1 Department of Philosophy, Stockholm University, Stockholm, Sweden 
arise in these theories. ${ }^{1}$ The presence of these objective probabilities indicates that an element of randomness-or stochasticity, ${ }^{2}$ as I will prefer to say-is an essential element in the theories' explanations and descriptions. ${ }^{3}$ My first point is that this stochasticity can be represented theoretically in three ways: in the determination of the system's initial conditions, in the evolution of its states, or in the realization of its physical properties. I then show that this furnishes a nice typology of familiar interpretations of statistical mechanics and of quantum mechanics, one where the apparent analogies between corresponding interpretations are made manifest.

Although, as said, there has been little written on these analogies in general, there is one well-known interpretational analogy which has been drawn out in some detail, namely, between (a certain popular version of) Bohmian mechanics and the so-called "Boltzmannian" approach to classical statistical mechanics (Allori et al. 2014; Allori and Zanghì 2004; Dürr 2001; Dürr et al. 1992, 1995; Goldstein 2001, 2012). The basis of this particular analogy has been identified by their proponents as ontological in character. In both theories the basic entities of the world are characterized as particles moving deterministically in space and time, just as in classical particle mechanics. However, each necessarily goes beyond classical particle mechanics by, inter alia, introducing probabilities (or likelihoods of some kind), via macroscopic states and quantum states, respectively. Since the underlying dynamics is deterministic in both cases, the only way that objective probabilistic indeterminism can enter these interpretive pictures is in the setting of initial conditions (or, equivalently, in the realization of a particular complete deterministic history of particle motions from the set of possible motions). Accordingly, in statistical mechanics one sees the invocation of an initial "past state" (Albert 2000) and, analogously, in quantum mechanics the positing of a "quantum equilibrium" initial state (Dürr et al. 1992), both of which serve this essential requirement.

While proponents of these interpretations tend to highlight the common "primitive ontology" as the origin of the analogy (Allori and Zanghì 2004), I suggest that one can equally well point to the common implementation of stochasticity in terms of

\footnotetext{
${ }^{1}$ Quantum statistical mechanics would seem to be an important separate case to consider, but I will not discuss it as such, essentially for the reasons given in Wallace (2014, §9) and Wallace (2016a, §7). To put the point briefly: a state in quantum statistical mechanics is nothing more than a special kind of quantum state. Thus, it is sufficient to discuss states and probabilities in quantum mechanics, for this general case subsumes quantum statistical mechanics. This is in contrast to the case of classical statistical mechanics, which is not just a special case of classical mechanics. Statistical mechanics involves probabilities essentially in a way that classical mechanics does not.

${ }^{2}$ The term "stochasticity" might suggest the idea of a stochastic state dynamics, as in the term "stochastic dynamics". To some extent this is what I intend, although I do intend it somewhat more broadly, indicating a particular source of process randomness, but where that source may be identified in different aspects of the theoretical apparatus. I choose to use it for two reasons. One, relying solely on the term "probability" would most likely have far more misleading connotations than "stochasticity", given the long-standing and complex philosophical discussions about the interpretation of probability. Two, my approach will make important use of an ontic interpretation of physical probabilities, as I explain in Section 3, in which case they do indicate a genuine element of stochasticity in the world.

${ }^{3}$ This stochasticity can be thought to exist either in the world itself, i.e., as genuinely ontically, or to arise merely through an agential posit (McCoy 2018a). In this paper I will prefer the former viewpoint for convenience, although nearly everything I say is consistent with the latter interpretation.
} 
the selection of initial conditions. It follows from taking this point of view-that the implementation of stochasticity grounds the analogy and not a common ontological picture-that the possible interpretive analogies between quantum mechanics and statistical mechanics do not end at Bohmian mechanics and Boltzmannian statistical mechanics.

There are several reasons to take up the interpretive framework proposed here. In the first place, it makes heretofore merely implicit analogies explicit and explains them. In the second, it opens up the landscape of interpretations that may be considered in classical statistical mechanics, a landscape which has been little explored so far. In the third, it suggests how one might take advantage of ideas developed in one theoretical context and apply them to the other. This is because having an explicit grounding for the analogies serves as a conceptual map, of sorts, between the two theories, which one can use to connect or translate interpretational issues between them. In some cases this might problematize some unexamined presuppositions on one side of the analogy; in others it might suggest novel solutions which were apparent on one side but not the other. ${ }^{4}$

To indicate some of the potentially fruitful possibilities afforded by this point of view, I will consider how two interpretive issues in quantum mechanics, the quantum measurement problem and primitive ontology, arise also in statistical mechanics by exploiting the analogies I draw. First, I show how debates on primitive ontology in quantum mechanics can be reprised in classical statistical mechanics when one makes use of the full menu of interpretive options suggested by my proposal. I focus on the particular problems presented in Belot (2011) to illustrate its application. Second, I show how certain presentations of the quantum measurement problem may be seen as essentially posing an interpretive question concerning how to conceive of the stochasticity of quantum mechanics. I do this by constructing an analog of one of these presentations (Maudlin 1995) in classical statistical mechanics and indicating that the possible solutions to the problem are precisely the possibilities identified by my framework. Note, however, that in neither case will I advance any particular resolution to these problems here, as this paper's focus is on developing the interpretive framework itself.

I begin in Section 2 with the aforementioned Boltzmann-Bohm analogy, as it is most familiar and will be a useful contrast for my approach. In Section 3 I motivate and introduce my interpretive framework, situating various interpretations of statistical mechanics and quantum mechanics within it along the way. In Section 4 I connect the discussion of primitive ontology in quantum mechanics to the interpretive framework of this paper. Then in Section 5 I suggest how one can generate a statistical mechanical measurement problem analogous to (a certain presentation of) the quantum measurement problem, to which each of the implementations of stochasticity offers a solution strategy. Finally, Section 6 is a brief conclusion.

\footnotetext{
${ }^{4}$ Callender (2007) offers one useful example of the latter case: by exploiting the aforementioned (ontological) analogy between Boltzmannian statistical mechanics and Bohmian mechanics, Callender applies ideas and strategies from the former to address the issue of how probabilities in the latter emerge and should be interpreted. This case will be discussed further in Section 2.
} 


\section{Boltzmannian-Bohmian interpretations of mechanics}

According to their supporters, the key idea behind Boltzmannian statistical mechanics and Bohmian quantum mechanics is that these theories are fundamentally about individual systems of microscopic entities (Allori et al. 2014; Allori and Zanghì 2004; Dürr 2001; Dürr et al. 1992, 1995; Goldstein 2001, 2012), usually assumed to be collections of particles. Whereas the standard "Gibbsian" techniques in statistical mechanics are often understood to invoke a fictitious ensemble of microscopic systems (Lavis 1977) and standard quantum mechanics a "wave function" in place of classical particles or fields, Boltzmannian and Bohmian mechanics are said to have a clear ontology of "local beables" (Bell 2004). This is said to be a virtue because, as a matter of method, "the first step in the construction of a physical theory is to establish what are the mathematical entities (particles, fields, strings,...) with which one intends to describe physical reality" (Allori and Zanghì 2004, 1744). Supposing that this is a sage piece of guidance, let us follow it to see where it leads. ${ }^{5}$

\subsection{Classical mechanics}

So let us begin with a mathematically precise description of the physical reality which these theories purport. ${ }^{6}$ This physical reality is essentially based on the description of physical reality given by classical particle mechanics. It is set within the classical conception of Euclidean space $\Sigma$ and time $\tau$ (where these are supplied with the appropriate mathematical description — set theory will be mostly sufficient for my purposes here). At each instant $t \in \tau$, every physical particle $i \in \mathbf{N}$ (for $n$ labeled particles) has some location $x_{(t, i)} \in \Sigma$ in space, which one may describe mathematically with a map $\tau \times \mathbf{N} \rightarrow \Sigma$. Thus, for a particular particle $i$ at time $t$ we have $(t, i) \mapsto x_{(t, i)}$.

If one models classical space and time as a four-dimensional spacetime $\Sigma^{\tau}$, then each particle traces out a continuous motion $\gamma_{i} \subset \Sigma^{\tau}$ (the image set of a curve with pre-image $\tau$ ) in spacetime (its worldline). ${ }^{7}$ The key idea behind this construction is that, with a particular motion $\gamma_{i}$ in one's hands, one has all the physical content related to an individual particle available (given relevant observable functions to represent its physical properties). So in the simple case of classical mechanics, this ontological starting point leads directly to the complete descriptive content of the theory.

Mechanics is not usually described in terms of this space-and-time picture, instead making use of the notion of a configuration $q=\left(x_{1}, x_{2}, \ldots, x_{N}\right)$, which specifies the

\footnotetext{
${ }^{5}$ Some readers who are very familiar with the theories discussed in this section may wish to skip ahead to Section 2.4 for its upshot. However, the following subsections are not intended to merely rehash standard material but to illustrate how the analogy between statistical mechanics and quantum mechanics develops by the lights of the Boltzmannian/Bohmian, how it leads to a common interpretive challenge, and how that challenge can be resolved.

${ }^{6}$ See Souriau (1997) for a more thorough presentation of the terms and concepts employed throughout these sections.

${ }^{7}$ This motion can be projected down onto space $\Sigma$ as a trajectory $\gamma_{i}$ parameterized by time.
} 
location $x_{i}$ of each particle $i$ in the abstract configuration space $Q=\Sigma^{N}$. With configuration space, we can re-express the map $\tau \times \mathbf{N} \rightarrow \Sigma$ as a new map $\tau \rightarrow Q=\Sigma^{N}$ (i.e., by currying). Thus, for a particular time $t$ we have $t \mapsto q_{t}=\left(x_{1}, x_{2}, \ldots, x_{N}\right)_{t}$. This move to configuration space is fully equivalent to the space-and-time picture; it is just a convenient redescription.

To describe motions based on configuration space we need to make use of the extended configuration space $Q^{\tau}$. Each individual motion $\gamma_{i}$ of each particle $i$ in spacetime can be incorporated into a single continuous motion $\zeta \subset Q^{\tau}$ in extended configuration space. ${ }^{8}$ Again, nothing is lost (and equally nothing is gained, save convenience) by moving to configuration space, as a motion $\zeta$ is sufficient to capture all the physical content related to a complete physical system, an entire classical system of particles (given relevant observable functions to represent their physical properties).

The establishment of physical reality has so far proceeded handsomely, and there is no reason to question the ontological starting point from a physical point of view. Still, while the collection of motions $\gamma_{i}$ or the single motion $\zeta$ is descriptively adequate, it is not yet explanatorily adequate, for we lack the dynamical underpinnings (which may or may not be part of reality, depending on one's point of view) required for a mechanical explanation. To incorporate these underpinnings into our description of physical reality, we need to specify not only of the position of each particle at a time but also its velocity (or momentum) for an initial value problem to be well-posed. Therefore, it is necessary to expand configuration space to incorporate the additional "degrees of freedom", constructing the (time-indexed) phase space $\Gamma_{t}$, which has twice the degrees of freedom of $Q$ : half of them are the positional degrees of freedom and half the velocital or momental degrees of freedom.

A deterministic dynamics for a specific system can then be represented as a oneparameter family of bijective maps $\phi_{t}: \Gamma_{t} \rightarrow U$, indexed by time $t$, where $U$ is the space of dynamically possible motions of the system in extended configuration space $Q^{\tau}$. These dynamical maps send elements of the phase space ("microstates") at a time $t,(q, p)_{t} \in \Gamma_{t}$, to the system's full motion $\zeta$ through extended configuration space $Q^{\tau}$. In the usual case the time subscript may be dropped, as the time-indexed phase spaces are isomorphic. Note also that we may obtain time evolution between instants by composing dynamical maps with inverse dynamical maps. For example, for the evolution of a state between time $t$ and $t^{\prime}$ we use the map $\phi_{t t^{\prime}}: \phi_{t^{\prime}}^{-1} \circ \phi_{t}$, so that $(q, p)_{t} \in \Gamma_{t} \mapsto\left(q^{\prime}, p^{\prime}\right)_{t^{\prime}} \in \Gamma_{t^{\prime}}$, or, when the instantaneous phase spaces are isomorphic, simply $(q, p) \mapsto\left(q^{\prime}, p^{\prime}\right)$.

Mathematically speaking, the picture of reality obtained by following these incremental steps, beginning with nothing more than space, time, and particles, is unquestionably elegant and powerful. Perhaps there is room to question the ontological grounds of the dynamics, which may seem obscure. Various philosophical strategies may be employed, however, to reduce, eliminate, or otherwise prescind from the ostensible ontological significance of dynamical laws. After all, the descriptive content is contained entirely in the kinematical motions, not the dynamics.

\footnotetext{
${ }^{8}$ This motion can also be projected down onto configuration space $Q$ as a trajectory $\zeta$ parameterized by time, just as particle motions can be projected down onto space $\Sigma$ and parameterized by time.
} 


\subsection{Bohmian and Boltzmannian addenda}

In Boltzmannian statistical mechanics one adds to the classical picture above first the notion of a macrostate. While microstates are characterized (as before) by certain microscopic properties of particles (position and either momentum or velocity), macrostates are introduced to represent various macroscopic properties (like pressure). In the Boltzmannian picture, the latter are realized, however, by microstates in the following sense: if $M$ is the collection of the macrostates of some system, then there is a projection map $\pi: \Gamma \rightarrow M$ that partitions the phase space $\Gamma$ into macroregions associated bijectively with the macrostates. Therefore, if one knows the macrostate of a system, then one does not automatically know its underlying microstate; however, given a microstate, the corresponding macrostate of the system is absolutely determined. Furthermore, the macrostate dynamics is entirely determined by the microstate dynamics in virtue of this projection relation: a "macrostate motion" $v$ through $M^{\tau}$ is given by the projected microstate dynamics $\pi \circ \phi_{t}$ (where I abuse terminology to allow $\pi$ to project from microscopic motions in $Q^{\tau}$ to macroscopic motions $M^{\tau}$ in the obvious way). Thus, much as in classical particle mechanics, a motion in an extended (microscopic) phase space is sufficient to capture all the descriptive content of a system described by Boltzmannian statistical mechanics. So far so good.

Bohmian mechanics also goes beyond the classical picture, adding quantum states in the form of quantum wave functions. These are particular $\left(L^{2}\right)$ complex-valued functions on configuration space $Q \rightarrow \mathbf{C}$. The wave function dynamics is given by the Schrödinger equation (which is not determined completely by the particle dynamics, as the macrostate dynamics is in Boltzmannian statistical mechanics). I treat the wave function dynamics as maps of the form $U_{t}: \mathcal{H} \rightarrow \mathcal{H}^{\tau}$ indexed by time $t$, where $\mathcal{H}$ is the Hilbert space of wave functions (ignoring overall phase factors). Importantly, the particle dynamics in Bohmian mechanics is not the same as the dynamics in classical particle mechanics. Instead, it depends only on the particle configuration (not the system's state in phase space) and on the wave function. Thus, the particle dynamics of Bohmian mechanics, usually represented by the so-called "guidance equation" (for the particles are said to be "guided" by the wave function), can be given by maps of the form $\phi_{t}^{\psi_{t}}: Q \rightarrow Q^{\tau}$, indexed by time $t$ and the quantum state $\psi_{t}$ at time $t$. Nevertheless, despite the differences, a motion $\zeta$ in the extended particle configuration space is sufficient to capture all the physical content of a system described by Bohmian mechanics, exactly as in classical particle mechanics.

Clearly, Boltzmannian statistical mechanics and Bohmian quantum mechanics share many salient features. First, the basic ontology of the interpretations, our starting point, is particles moving in space and time. But, importantly, they also include some extra structure: macroscopic states or quantum wave functions (which may or may not be part of the basic ontology, depending on how these are interpreted (Ney and Albert 2013)). Still, since a motion in extended configuration space is sufficient to capture the physical content of a system described by Bohmian mechanics, Boltzmannian statistical mechanics, or classical mechanics, one might well say, with Bell, that "in physics the only observations we must consider are position observations, if only the positions of instrument pointers" (Bell 1982, 996). Second, the dynamics 
of the particles and of the extra ontology are fully deterministic. Therefore, the core of the two theories is essentially that of classical mechanics, with only slight modifications needed to account, respectively, for certain macroscopic phenomena (temperature, the approach to equilibrium, etc.) or quantum phenomena (spin, entanglement, etc.). ${ }^{9}$ That this common picture of microscopic entities moving in space and time is applicable in such varied theories is, one must admit, surely a point in its favor.

\subsection{Probability}

However, this picture is still incomplete in the cases of statistical mechanics and of quantum mechanics, since both theories depend essentially on probabilities for their empirical content, and this is captured principally by statistics of observables. In statistical mechanics the relevant empirical frequencies are of the macroscopic properties; in Bohmian mechanics they are of quantum observables (that are, according to Bohmian doctrine, of course, ultimately reducible to particle positions). Thus we must account for probabilities in our theoretical description in addition to everything that has come so far. Probabilities are implemented in the theory in somewhat different ways, but the statistics come out looking very similar, as we will now see.

In Boltzmannian statistical mechanics, probability distributions are associated with macrostates (with finite-measure macroregions of the microscopic phase space). The initial probability distribution $\rho_{0}$ (on phase space $\Gamma$ ) of a system in an initial macrostate $M_{0}$ is stipulated to be uniform with respect to the Liouville measure $\mu$ associated with the underlying phase space and to have this uniform support only on the macroregion $\pi^{-1} M_{0}$ (identified with the macrostate $M_{0}$ ). Since the probability distributions are defined on phase space (they are maps $\Gamma \rightarrow[0,1]$ ), the dynamics of these probability distributions (the Liouville dynamics) is fully determined by the deterministic particle dynamics. Thus, the probability distribution at time $t, \rho_{t}$, is given by $\rho_{0} \circ \phi_{t 0}$. In effect, one pushes $\rho_{0}$ forward from $\Gamma_{0}$ to $\Gamma_{t}$ using the dynamical maps for the microstates.

In statistical mechanics, the empirically relevant observables are macroscopic observables, since it is presumed that microstates are epistemically (or at least practically) inaccessible. These should naturally be defined on macrostates, but it is just as well to define them on phase space (since one could easily pull macroscopic observables defined on the macrostates back onto phase space with the projection map $\pi$ ). Thus, we may treat any observable $\mathcal{O}$ as a map $\Gamma \rightarrow \mathbf{R}$, just as in classical mechanics, and use it to calculate statistics. For example, the expectation value of $\mathcal{O}$ at time $t$ for a system that started in an initial macrostate with associated probability distribution $\rho_{0}$ is given by the following formula:

$$
\langle\mathcal{O}\rangle_{\rho_{t}}=\int_{\Gamma} \rho_{t} \mathcal{O} \mathrm{d} \mu
$$

\footnotetext{
${ }^{9}$ In order to capture novel quantum phenomena (like spin), Bohmian mechanics must be able to capture spin observables in terms of position observables. See Daumer et al. (1996), Goldstein (2017, §11), or Dürr and Teufel $(2009, \S 8.4)$ for the Bohmian resolution.
} 
As one can see, the expectation value essentially sums together the values of the observable $\mathcal{O}$ in each microstate in $\Gamma$, weighted by the probability assigned to that microstate by $\rho_{t}$.

In quantum mechanics, the wave function, while certainly no probability distribution in and of itself, is nevertheless a proxy of sorts for the probabilistic content of the theory. Bohmian mechanics, which privileges the position basis, and, hence, the wave function, does permit the identification of an initial probability distribution in "quantum equilibrium" (Dürr et al. 1992). This means that the initial probability distribution $\rho_{0}$ (on configuration space) is such that the probability of a particular initial configuration is given by the squared amplitude of the initial wave function: $\rho_{0}=\left|\psi_{0}\right|^{2}$. In this case the guidance equation consistently gives the dynamics of $\rho_{0}$, so that one can write the probability distribution at time $t$ as $\rho_{t}=\rho_{0} \circ \phi_{t 0}^{\psi_{0}}{ }^{10}$

Quantum mechanical observables (like position $q$ ) are standardly represented as self-adjoint operators $\mathcal{O}: \mathcal{H} \rightarrow \mathcal{H}$ on the space of quantum states. In Bohmian mechanics, quantum observables are, again, understood to be ultimately reducible to position measurements, so they are not to be interpreted "realistically" (Daumer et al. 1996). Nevertheless, their formal use is unobjectionable to the Bohmian. Still, we may simplify our formalism by preferring the position basis and writing observables as functions of particle configurations: $\mathcal{O}: \mathcal{Q} \rightarrow \mathbf{R}$. The expectation value for an observable $\mathcal{O}$ in Bohmian mechanics then closely resembles the expectation value in statistical mechanics:

$$
\langle\mathcal{O}\rangle_{\rho_{t}}=\int_{Q} \rho_{t} \mathcal{O} \mathrm{d} q .
$$

Thus the analogy which began with the theories' primitive ontology continues all the way to their empirical content (which takes the form of statistics of observables).

\subsection{Deterministic probabilities}

It is here, though, that the purported picture of reality wavers somewhat. Trying to assimilate the probabilistic posits of the previous section into theories that aim to maintain a classical ontic picture of deterministic particle mechanics leads to a significant philosophical problem in interpreting these probabilities. ${ }^{11}$ In brief, it is as follows. On the one hand, if the particle configurations determine all observable properties, what can probability represent other than a measure of uncertainty over what the actual configurations are? On the other hand, it cannot be mere uncertainty, since the empirical content of the theories is entirely contained in statistics of observables and, hence, is determined by the probability distribution one uses to describe a system-these probability distributions, in other words, have empirical significance. In statistical mechanics such conflicting concerns have been raised in the context of

\footnotetext{
${ }^{10}$ By "equivariance", the probability distribution $\rho_{t}$ at time $t$, which is determined by pushing forward the particle dynamics flow onto the space of probability distributions, will also be equal to $\left|\psi_{t}\right|^{2}=\left|U_{0 t} \psi_{0}\right|^{2}$, as determined by the wave function dynamics $U_{0 t}=U_{t}^{-1} \circ U_{0}$. Cf. (Dürr et al. 1992, 855).

${ }^{11}$ See McCoy (2018a) for a more complete discussion, on which this section is based.
} 
discussions over the "paradox of deterministic probabilities" (Loewer 2001; Winsberg 2008; Lyon 2011). Bohmian mechanics, given the relevantly shared structure, faces the same concern (Callender 2007).

Many philosophers interested in the question of how to interpret such physical probabilities have been motivated by the "Humean" approach to laws and chances popularized by Lewis $(1973,1981,1983) .{ }^{12}$ The Humean is skeptical of strong ontological interpretations of laws, chances, and other modal connections which would inflate ontology beyond simple, familiar, actual entities (particles moving around in space and time being a particular favorite). Although Humeans accept that laws, chances, etc. play an important explanatory role in science, they argue that these things objectively reduce to their preferred ontology.

To avoid the potential conflict between the determinism of the dynamical laws and any indeterminism arising from statistical mechanical probabilities, Loewer (2001) chooses to adopt just such a Humean approach in his prominent solution, in which he proposes that probabilities be assigned to possible microscopic initial conditions. That is, there are (Humean) chances for a system (or the whole universe) to begin in the various initial microstates, after which the actual initial microstate's evolution is deterministic ever after. The Humean, of course, does not believe that there was an actual "chancy" event which brought about the initial state of the system (or universe). A Humean of Loewer's stripe only claims that the best, objective systematization of the actual, occurrent facts about the world allows one to describe the world theoretically in just this way. Evidently, though, setting such Humean scruples aside, one can give a realist interpretation of these chances as well Demarest (2016).

As it happens, a similar solution to this problem is offered by Dürr et al. (1992) in the context of quantum mechanics, by way of the already mentioned "quantum equilibrium". Although their preferred story differs in detail, ${ }^{13}$ Loewer's Humean account might well be applied here too, by supposing that the initial chances for the particles of the system (or universe) to begin in the possible initial configurations are given by $|\psi|^{2}$, the norm squared of its wave function. ${ }^{14}$

\subsection{Reflections}

Having seen the analogies between Boltzmannian statistical mechanics and Bohmian quantum mechanics drawn out in detail, let us now reflect on where following the methodological advice with which we began has led us. From common ontological assumptions we were led to a common interpretive challenge and then to similar approaches to resolving it. Indeed, the shared mathematical and physical

\footnotetext{
${ }^{12}$ This stance has been defended by Loewer $(1996,2001,2004)$ and, in an alternative version, by (Frigg and Hoefer 2015) in the context of statistical mechanics.

${ }^{13}$ Dürr et al.'s $(1992,1995)$ view relies on the notion of "typicality" (which Goldstein $(2001,2012)$ also invokes in statistical mechanics) rather than probabilities. As I find the available criticisms of their use of typicality convincing (Frigg 2009; Luczak 2016), I will not discuss the notion here.

${ }^{14}$ Although, again, one does not have to believe, in a Humean spirit, that there was such an actual chancy beginning, one may do so (Callender and Weingard 1997).
} 
structure of the two theories makes this seemingly inevitable. As said, the BohmianBoltzmannians emphasize the shared structure that comes from common ontological assumptions as the basis of the analogies between the two theories, and they take this "clear" ontological starting point as a point in their favor. ${ }^{15}$ But given the significant philosophical maneuvering required to make sense of the probabilities introduced by statistical mechanics and quantum mechanics, this appears to require taking on quite a lot of "quantum philosophy" with one's "quantum physics".

While I find the inherent philosophical tensions in such an approach interesting, what is immediately relevant for my purposes is that a different common assumption has emerged through efforts to avoid the potential conflict with determinism. That assumption was that the source of all objective probabilities is an initial assignment of probabilities to initial configurations or states. What if we take this idea as the source of the analogy instead? Let us suppose that as a first step in the interpretation of a probabilistic physical theory we establish where stochasticity enters into physical reality. Then, by taking what is stochastic about the theory to be the selection of initial conditions, we might realize that this interpretation is precisely one which allows for a microscopic ontology of deterministically evolving classical particlesshould one like to "insist that 'particles' means particles" (Dürr et al. 1995, 137). I will pursue the idea behind this starting point in the next section by developing three alternative implementations of stochasticity, which will show that the particular way that Boltzmannians and Bohmians interpret their theories is merely one particular choice of how to characterize the probabilistic content of statistical mechanics and quantum mechanics.

\section{Stochasticity in physics}

In interpreting classical statistical mechanics and quantum mechanics, we should recognize as a first step that these are theories of statistical physics: their predictive empirical content depends essentially on probabilities and is captured in the form of statistics (Wallace 2015). This is in contrast to other physical theories, like particle mechanics, general relativity, and thermodynamics, whose empirical content is not essentially statistical and does not rely essentially on probabilities. This difference tells us that probabilities play an epistemically special role in statistical physics. Philosophically, one would like to understand how to speak about the world such that the empirical content of the theory takes this form.

As this task depends on understanding how probabilities are involved in generating empirical content, much of the distinctive interpretational challenge will concern understanding the nature of probability. This is a notoriously vexed subject in philosophy, and my approach cannot hope to comprehend all the possibilities that have been considered. My strategy is instead to presume a generically ontic interpretation of physical probabilities in this paper.

\footnotetext{
${ }^{15}$ For my part, I doubt point particles and other such "mathematical entities" are somehow clearly concrete objects of experience, pace Dürr et al. (1995). But the reader is invited to consult his or her own perceptual faculties.
} 
What I mean by an ontic interpretation of physical probabilities is that the probabilities reflect some element of randomness or chanciness in the world. This presumption makes it easiest to demonstrate the interpretive analogies I see between statistical mechanics and quantum mechanics. It also makes the probabilities of the theories trivially objective, something which can be difficult to obtain on other interpretations. ${ }^{16}$ An ontic interpretation is simple and clear: it straightforwardly presumes that probabilities represent real worldly structure, much like other formal features of physical theories are taken to represent real worldly structure. Of course, one can always raise skeptical challenges to this presumption and alternative interpretations, but indulging in them here would only be a distraction. Nevertheless, I am also not endorsing any particular interpretive position about the nature of probabilities by employing an ontic manner of speaking about probabilities; much less do I commit to any further metaphysical characterization of ontic probabilities in terms of dispositions, propensities, or whatnot.

Given an ontic interpretation of probabilities in statistical physics, I suggest that there are only a few distinct options available for implementing genuine chances in quantum mechanics and statistical mechanics. To see how these three options are suggested, we need to look at how the empirical content of the theories is represented. This is, again, in the form of statistics. The simplest statistical moment, the mean or expectation value, will be sufficient to demonstrate the point.

In classical statistical mechanics, the statistical state of a system is conveniently taken to be represented by a probability distribution $\rho: \Gamma \rightarrow[0,1]$ on phase space $\Gamma$. One can construct the corresponding probability measure out of it by making use of the natural Liouville measure $\mathrm{d} \mu$ associated with the phase space. ${ }^{17}$ The expectation value of an observable $\mathcal{O}$ when the system is in state $\rho$ is then

$$
\langle\mathcal{O}\rangle_{\rho}=\int_{\Gamma} \rho \mathcal{O} \mathrm{d} \mu
$$

where the integral is over all phase space points $x \in \Gamma$.

For the moment, I enjoin resistance against the familiar interpretation of the points $x \in \Gamma$ as microscopic states with ontic significance-especially as configurations

\footnotetext{
${ }^{16} \mathrm{~A}$ referee suggests relaxing my presumption from ontic to merely objective. I take it that what makes probabilities objective is that they are determined in some way by facts about the world. Ontic probabilities, or chances, are objective because such probabilities are grounded in chancy features of the world. Other kinds of probability may arguably also be objective. For example, frequentist probabilities are determined by facts about the world, namely frequencies, and so are Humean chances; probabilities also may be grounded by ergodic facts about temporal behavior or through the method of arbitrary functions. Plainly, these accounts of non-ontic objective probability (epistemic probability) are a motley bunch, and they do not generally hook into the formalism of statistical physics neatly (they add in epistemic considerations, after all). For this reason, presuming probability to be merely objective would obscure the points I make in this paper, which are clearest on an ontic interpretation, where one conceives the formalism as more directly representational than one does on a non-ontic interpretation. I relate my views on these various kinds of probability in more detail in McCoy (2018a).

${ }^{17}$ One might equally well take the probability measure $P$, so constructed, as the statistical state. A probability measure $P$ associated with a phase space $\Gamma$ is a map $\mathcal{L} \rightarrow[0,1]$, that is, from Lebesgue-measurable subsets $\mathcal{L}$ of $\Gamma$ to the real-number interval $[0,1]$, which satisfies the usual probability axioms. It could also be defined in terms of the probability distribution for Lebesgue-measurable sets $U \in \mathcal{L}$ by $U \mapsto \int_{U} \rho \mathrm{d} \mu$.
} 
of particles with specific momenta. To make this interpretation less inviting, we may confine our attention to a particular observable $\mathcal{O}: \Gamma \rightarrow \mathbf{R}$ and look at the probability space associated with its image set $\mathcal{O}[\Gamma]$ (McCoy 2018b): The Lebesgue measurable sets $\mathcal{L}$ may be pushed forward onto $\mathcal{O}[\Gamma]$ using $\mathcal{O}$; I denote this collection of subsets as $\mathcal{L}_{\mathcal{O}}$. The statistical state $\rho$ can be pushed forward onto $\mathcal{O}[\Gamma]$ as well using $\mathcal{O}$; the probability distribution $\rho_{\mathcal{O}}$ is therefore $\mathcal{O}_{*} \rho$. With these three pieces, $\mathcal{O}[\Gamma], \mathcal{L}_{\mathcal{O}}$, and $\rho_{\mathcal{O}}$, we have defined a probability space. The Lebesgue measure $\mathrm{d} \omega$ on $\mathbf{R}$ is a natural standard of integration which we could use to write an expectation value formula like (3), but for future convenience I will incorporate the probability distribution into a new integration measure $\mathrm{d} \mathcal{O}_{\rho}$ by defining it as $\rho_{\mathcal{O}} \mathrm{d} \omega$. In this case one may write the expectation value of any particular observable $\mathcal{O}$ as

$$
\langle\mathcal{O}\rangle_{\rho_{\mathcal{O}}}=\int_{\mathcal{O}[\Gamma]} \omega \mathrm{d} \mathcal{O}_{\rho},
$$

where $\omega \in \mathcal{O}[\Gamma]$ is a particular observable outcome. By this procedure we have quined away the microstates of phase space, with the additional benefit of making the theory's empirical content manifest solely in terms of observable outcomes $(\omega)$ and (proxies for) statistical states $\left(\mathrm{d} \mathcal{O}_{\rho}\right)$.

While this formal redescription may aid in the avoidance of the premature reification of microstates, it more importantly reveals a key formal similarity to expectation values in quantum mechanics. Taking the simple case of projection-valued measures, if we let $\mathcal{O}$ be a self-adjoint operator on the Hilbert space $\mathcal{H}$ and $\psi$ be a particular pure quantum state, then the probability distribution $\rho_{\mathcal{O}}$ on the set of possible observable outcomes $\sigma(\mathcal{O})$ (the spectrum of $\mathcal{O}$ ) is determined by the Born rule: $\rho: \omega \mapsto\left\langle\psi, \mathcal{P}_{\omega} \psi\right\rangle$, the inner product of $\psi$ and the projection operator $\mathcal{P}_{\omega}$ (that projects onto the eigenspace associated with $\omega$ ) applied to $\psi$. Now, let $\mathrm{d} \mathcal{O}_{\rho}$ be the integration measure incorporating the probability distribution $\rho_{\mathcal{O}}$. Then the quantum expectation value is expressible essentially just as in (4):

$$
\langle\mathcal{O}\rangle_{\rho_{\mathcal{O}}}=\int_{\mathcal{O}[\mathcal{H}]} \omega \mathrm{d} \mathcal{O}_{\rho} .
$$

In formulas like this, orthodox quantum mechanics (Wallace 2016b) transparently supplies us with the empirical content of the theory. Moreover, in orthodox interpretations of quantum mechanics there are, of course, no underlying "microscopic states" (let alone states interpreted in terms of particles). There are just quantum states represented by vectors in Hilbert space. Although presenting statistical mechanics' empirical content in a similar form, solely in terms of observables and statistical states, is somewhat unorthodox, it does serve to neatly highlight the theories' shared structure used to represent their empirical content. From this ontologically neutral point, one can then freely ask further questions about unexpressed representational structure, without ontological prejudice. ${ }^{18}$

\footnotetext{
${ }^{18}$ This is all the clearer when the theories are formulated in terms of operator algebras, but the technical complexities of formulating statistical theories in these terms would be too much a digression here. For the relevant perspective, see, e.g., Landsman (2017) and Rédei and Summers (2007).
} 
Time evolution must also be incorporated into the picture we have so far. Let us assume that the states, or rather our proxies for states, the probabilistic integration measure $\mathrm{d} \mathcal{O}_{\rho}$, evolve in time rather than the operators. I defer saying more about whether the dynamics is deterministic or indeterministic and how it operates for the moment. In any such case, since I assume that the observables do not change over time, any operation of the dynamics can only affect the integration measure. To mark its time-dependence, therefore, I will add a superscripted $t$ to it as a reminder:

$$
\langle\mathcal{O}\rangle_{\rho}^{t}=\int_{\mathcal{O}[\cdot]} \omega \mathrm{d} \mathcal{O}_{\rho}^{t} .
$$

Thus, the empirical content of statistical physics, here demonstrated by example in the form of an expectation value, is generated by observables, states, and dynamics (where the latter two have been combined in the integration measure). By merely inspecting how their empirical content is formulated, it is apparent that there are essentially three possible ways to conceive of chances operating in statistical physics - three possible interpretations of stochasticity. These are (1) that initial states are chancy, (2) that the operation of the dynamics is chancy, and (3) that the realization of (observable) properties themselves is chancy. ${ }^{19}$ Let us consider each of these in turn.

First, if one supposes that what is random about a physical system (as described by these two theories) is its initial conditions and nothing else, then it follows that every observable property $\mathcal{O}$ of the system has a value determined by the outcome of this random trial for all times ever after. Nothing else is left to chance but this initial random trial or chance event, and the chances for each outcome are given by an initial probability distribution $\rho_{0}$. If follows that the use of statistical states at times other than the initial one (such as expectation values like (6)) must be essentially epistemic in nature: they represent uncertainty over the actual realized outcome of the trial. Importantly, this interpretation mandates characterizing the deterministic evolution of the system after the initial random trial by introducing "hidden variables". These actual (hidden variable) states can be used to construct a deterministic dynamics for all times (except for the initial time, where the state is indeterminstically fixed by a random trial) and can represent the system's actual properties via an ontological interpretation (in terms of particles or whatnot).

This interpretation of stochasticity is the one found in the specific interpretations of statistical mechanics and quantum mechanics discussed in the previous section.

\footnotetext{
${ }^{19}$ Other authors have made similar claims about how many kinds of probability there are in physics, but as far as I am aware none has offered a typology like I have and none has provided any rationale for their proposal. For example, Maudlin (2007b, 2011) claims that there are three kinds of objective probability: stochastic dynamics, Humean chance, and deterministic chance. Given a distinction between objective probabilities and ontic probabilities (chances), these categories are clearly not exhaustive: they are merely three (popular) examples of objective probability. The first is a kind of ontic probability, the second might be a kind of ontic probability, depending on how understands Humean chances, and the third is a kind of epistemic probability.
} 
In Boltzmannian statistical mechanics the hidden variable are classical mechanical states (usually interpreted as particle states), and in Bohmian quantum mechanics they are configurations of local beables (particles or whatnot). The outcome of the initial chance event, then, is a particular initial classical state or configuration of beables. This state evolves deterministically and at every time all observable properties of the system are determined by it. As observers, we assume that we know the initial probabilities associated with the initial states $\left(\rho_{0}\right)$ but not the initial microstate. With $\rho_{0}$, however, we can calculate statistics for the system's observables since we know how it evolves in time (via the deterministic dynamics). In this way, the "initial chance" story makes sense of all the formalism introduced in the previous section (recognizing, of course, that this story may be modified by fictionalizing or otherwise eschewing the reality of initial chances, as in the Humean chance account).

Second, if one supposes that what is random about a physical system is solely its state transitions (the operation of its dynamics), then there are (at least some) state transitions that are not completely determined by the sequence of preceding states. That is, at least some of the evolution of the probability distribution incorporated into the expectation value formula is not determined. In such interpretations involving dynamical stochasticity, expectation values and the other statistical moments determined by the state are the complete predictable empirical content of the system. Of course, in the wake of state transitions (whether indeterministic or deterministic), a history of observable outcomes is generated by the system's evolution. However, as this generated history is to some extent subject to randomness, one cannot expect exact agreement between the generated histories of observable outcomes and the expectations generated by the statistical predictions (although one does of course expect statistical agreement).

There are many ways indeterministic evolution could be implemented with stochastic dynamics. ${ }^{20}$ Accordingly, there are several examples of this kind of interpretation of stochasticity in quantum mechanics and statistical mechanics. Although the details of how stochastic mechanisms are incorporated into dynamics vary between quantum mechanics and statistical mechanics (and even within the theories), it is not worth examining them in all their details here; my aim is simply to capture what they have in common and mention a few examples for illustration.

In quantum mechanics, approaches with indeterministic dynamics are generically called collapse theories (Ghirardi 2016). The traditional Copenhagen interpretation invokes wave function collapses during measurement; insofar as it is an interpretation of quantum mechanics, it is a collapse theory. The reader may well be acquainted with more rigorous collapse theories. For example, in the Ghirardi-Rimini-Weber (GRW) approach, quantum states evolve according to the Schrödinger equation most of the time, but the state of the system occasionally experiences indeterministic "collapses"

\footnotetext{
${ }^{20}$ Note that one might claim that the initial chances interpretation is strictly speaking a special case of this interpretation, one with all the indeterminism effected at an initial time, but I will keep them separate here.
} 
to different quantum states. Other versions allow for continuously indeterministic evolution (e.g., Pearle's continuous spontaneous localization). ${ }^{21}$

It is worth noting that in all familiar cases the collapse dynamics is implemented solely on the level of the quantum state. Nevertheless, the stochastic dynamics account can be supplied with "hidden variables" which can then be characterized ontically, just as in the initial chance approach. The dynamics of the statistical states in this case will be given in terms of the microscopic dynamics, and the observable properties of the system can be determined by the hidden variables. This is actually the usual way that stochastic dynamics is implemented in classical statistical mechanics (Wallace 2014; Luczak 2016). ${ }^{22}$ Indeed, many of the most well-known equations from non-equilibrium statistical mechanics implement the dynamics directly on microstates, such as the Langevin equation; others, like the Fokker-Planck equation, implement a dynamics on the level of the statistical state (but are generally constructed out of a microscopic dynamics). One is not, however, forced to posit classical microstructure in non-equilibrium statistical mechanics; one could, that is, follow the example of quantum mechanics and take, say, the Fokker-Planck equation at face value as a dynamics of statistical states.

Third, and finally, if one supposes that what is random about a physical system is solely its observable properties, then it follows that state transitions must be deterministic. That is, while the evolution of the statistical state $\rho$ is deterministic, the realization of actual properties of the system is stochastic. This is contrast to the previous case, where the evolution of the statistical state is stochastic, and the realization of actual properties is fully determined by the state. In both cases, however, it is because of the physical indeterminism present in the system that statistical moments determined by the state are the complete predictable empirical content of the system. Again, a history of observable outcomes is generated by the physical stochastic process, but this may not be a perfect match with the expectations generated by statistical predictions based on the statistical state.

Before saying more about this interpretation of stochasticity, it is useful to contrast the second and third interpretations with classical mechanics. In classical mechanics, the state dynamics is deterministic and states are individuated by the properties of the system. That is, the states determine the observable properties. Thus there is a "double determinism" in classical mechanics. The indeterminism of a probabilistic physical theory like classical statistical mechanics or quantum mechanics (on

\footnotetext{
${ }^{21}$ Are these not simply alternative theories to "normal" quantum mechanics, "since they actually are (in principle) empirically different from the standard theory" (Ghirardi 2016), rather than mere variants of a particular interpretation of stochasticity? But note that they may also be fashioned so as to not make any novel empirical prediction at all, for example, by making their indeterministic dynamics mimic as closely as possible the deterministic state dynamics of the Schrödinger equation. Indeed, as Werndl (2009, 2011) shows, one can always find some such observational equivalence between deterministic and indeterministic theories. As the empirically adequate collapse theories appear to be in the latter camp at present (Ghirardi 2016), the question of whether collapse theories are theories or interpretations is somewhat moot. In any case, since my aim is not to assess the future of quantum mechanics but interpret the quantum mechanics we have, I treat collapse theories as interpretations here.

${ }^{22}$ There is, however, a variety of approaches and techniques utilized in non-equilibrium statistical mechanics. Unfortunately, there remains very little philosophical work on non-equilibrium statistical mechanics. Sklar (1993) and Uffink $(2007, \S 7)$ give selective summaries.
} 
an ontic interpretation) means that (at least) one of these two roles of state must become indeterministic. In the stochastic dynamics interpretation, the state dynamics is indeterministic, while the observable outcomes remain fully determined by the state. In other words, observables are not random variables, states are. In the stochastic observables interpretation, the state dynamics is deterministic, but the observable outcomes are random. In other words, observables are true random variables, states are not.

This third interpretive possibility has seldom been acknowledged in the literature, although there has been some recent discussion (McCoy 2018b; Frigg and Werndl 2018) in the context of classical statistical mechanics. There are several older approaches that appear to conform roughly to the basic idea of deterministic state dynamics with stochastic observables, such as those programs that Uffink (2007) calls "statistical thermodynamics"; also, some aspects of Prigogine's or Khinchin's approach agree with the interpretive philosophy given here, especially in their rejection of classical microstates. ${ }^{23}$ Frigg and Werndl (2018) also suggest several ways in which Gibbs' approach to equilibrium statistical mechanics might be interpreted, one of which (that they call "bare probabilism') is perhaps naturally characterized as a stochastic observables interpretation. ${ }^{24}$

It is less obvious which interpretations of quantum mechanics are compatible with this way of conceiving of probabilities. There is at least one popular candidate which might be thought of as having stochastic observables, that is, were its quantum probabilities understood as chances. This is the many worlds interpretation (or, perhaps better, the more general "orthodox quantum mechanics" discussed by Wallace 2016b). I say this because many worlds quantum mechanics eschews a primitive ontology of deterministically evolving particles, and it maintains a deterministic state dynamics at all times. In this way it is similar to the bare probabilism of Frigg and Werndl (although one must acknowledge the fact that quantum probability is a far more subtle matter to handle than classical probability). That said, most proponents of the many worlds interpretation do not attempt to give an ontic interpretation to quantum probabilities at all, instead favoring, for example, a "functional" interpretation (Saunders 2010; Wallace 2012) or an "epistemic" interpretation (Sebens and Carroll 2016). What probabilities in many worlds quantum mechanics are has, indeed, been a matter of much debate (Greaves 2007), and it would be too much of a diversion to enter into the debate here, much less propose a novel interpretation of quantum probabilities along these lines. So I do not mean to push the case that many worlds quantum mechanic is in the general category of stochastic observables very far here. In any case, other ideas, for example, an "ensemble" interpretation of quantum mechanics (Ballentine 1970) or, perhaps, the consistent histories approach (Griffiths 2019), might also be seen as falling under the stochastic observables interpretation when supplied with an ontic interpretation of probability, especially insofar as they share relevant features of the approaches to statistical mechanics mentioned in the previous paragraph.

\footnotetext{
${ }^{23}$ See, e.g., discussion in Sklar (1993), where such proposals are termed "radical" and "revisionist" interpretations of statistical mechanics.

${ }^{24}$ My own proposal of such an interpretation is presented and defended in McCoy (2018b).
} 
These, then, are three ways of conceiving of the stochasticity of statistical physics. They are most easily seen by assuming an ontic interpretation of probability, which is why I chose to do so, but their usefulness as categories can be extended to other interpretations of such theories which identify probabilities as merely epistemic. Furthermore, the different starting point of this section, compared to the previous one, is noteworthy, as it should now be clear that strong assumptions about the primitive ontology of the theories can easily obscure significant alternative possibilities. It is for this reason that I began by looking at the statistics which account for the empirical content of such theories. These can be cast in a suggestive form by making use of the common dynamical structures of statistical mechanics and quantum mechanics. As we observed, stochasticity can be associated, on the face of it, with one of three different components. These three components were the initial conditions, the dynamics, and the observables of a physical system.

A choice of one of these then suggested a particular interpretation (or class of interpretations) of statistical mechanics and of quantum mechanics. The first leads to, for example, Boltzmannian statistical mechanics and Bohmian quantum mechanics, the analogy between which was described in the previous section. Whereas there I began as the Boltzmannian/Bohmians prefer, with the common ontological posit of particles moving in space and time, I argued in this section that it is also possible to view the analogy between the two theories as coming from the choice of an interpretation of stochasticity. The second choice of where to locate stochasticity leads to another clear analogy, namely between the variety of approaches termed stochastic dynamics in statistical mechanics and the variety of collapse theories in quantum mechanics. The third choice leads to what I have been calling the stochastic observables interpretation. Although the link of this interpretation with well-established approaches in statistical mechanics and quantum mechanics is less developed, I believe that Gibbsian statistical mechanics and orthodox quantum mechanics serve ably as suggestive, albeit under-developed, examples. Serious interpretive work is required for this suggestion to be realized and adequately justified however.

\section{Primitive ontology}

The framework for connecting interpretations of quantum mechanics and statistical mechanics with one another which I developed in the previous section makes it possible to see how interpretive problems and solutions can be portable from one theory to the other. In this section and the next, I give two explicit examples where this may be done. In this section I export the present debate concerning "primitive ontology" in quantum mechanics (Belot 2011; Ney and Phillips 2013; Allori et al. 2014; Esfeld 2014) to classical statistical mechanics. A detailed discussion of this debate and its importation into statistical mechanics would be a topic in its own right; my aim is not to survey the entirety of this debate or press for one view or another. It is to show how substantially the same issue concerning primitive ontology arises in statistical mechanics, once one recognizes that similar kinds of interpretation are available there as in quantum mechanics — due to the interpretational analogies I have developed. 
To this end I focus only on two specific problems related to primitive ontology raised by Belot (2011): what he calls the "macro object problem" and what I will call the "extra ontology problem". An interpretation of quantum mechanics that specifies properties possessed by regions of spacetime has come to be called a primitive ontology. The version of Bohmian mechanics described above, according to which the material ontology of a quantum system consists in particles (Esfeld et al. 2014), has a primitive ontology, as do collapse interpretations (Maudlin 2007a) that include an ontology of "flashes" (Esfeld and Gisin 2014) or "matter densities" (Egg and Esfeld 2015). If one supposes that quantum physics provides a fundamental description of reality, then, according to proponents of a primitive ontology, it should describe a world of stuff and things moving around in space and time. Interpretations of quantum mechanics that suppose that the basic quantum description of the world is in terms of the quantum state (perhaps out of which macroscopic reality emerges) lack a primitive ontology. Among these views are wave function realism (Albert 2013; North 2013) and spacetime state realism (Wallace and Timpson 2010).

That there has been no such analogous discussion in statistical mechanics is presumably owed to the issue being regarded as long settled. Insofar as one demands a realist interpretation of statistical mechanics, its ontology is thought to consist of one class of entity: classical entities like particles (which evolve deterministically). I have argued, however, that the different interpretations of stochasticity imply that there are interpretations of statistical mechanics analogous to the interpretations of quantum mechanics, ones which indeed may not reduce to this specific ontology. One can therefore ask what the ontology of these former interpretations is, as well as how the ontologies of the two theories, statistical mechanics and quantum mechanics, relate.

Now to the two problems mentioned above. First, Belot's "macro object problem" poses the challenge of explaining how quantum mechanics provides truth conditions for the assignment of properties to ordinary macroscopic objects. If macroscopic objects are simply composed of microscopic objects and their properties are entirely reducible to the properties of the latter, then interpretations which adopt a primitive ontology, like Bohmian mechanics, would seem to have an important advantage in solving the macro object problem. The specification of microscopic properties in spacetime, after all, is (in the best case) just one relation away from specifying macroscopic properties in spacetime. Interpretations of quantum mechanics without a primitive ontology, by contrast, must furnish an evidently more complicated story for how macroscopic objects possess the properties that they appear to possess.

Let us try to pose a macro object problem in classical statistical mechanics. How does this theory provide truth conditions for the assignment of properties to ordinary macroscopic objects? The usual answer is that macroscopic objects (boxes of gas) are simply composed of microscopic classical mechanical objects (molecules of gas, say) and their properties are entirely reducible to the latter. To give this answer, however, one must supplement the statistical state with an actual microstate. Just as collapse interpretations and Bohmian mechanics possess a primitive ontology in quantum mechanics which can address the macro object problem, so too, it seems, can stochastic dynamics and Boltzmannian approaches be described as having a primitive ontology, one which makes a significant start towards addressing the 
macro object problem in this context. Evidently, other interpretations of statistical mechanics without a primitive ontology will have to furnish a more complicated story in order to address the problem. That is not to say, of course, that some such story cannot be given, just as it is not to say so in quantum mechanics.

Second, Belot challenges primitive ontologists in quantum mechanics to make sense (ontologically speaking) of the quantum state. He claims that their interpretations are dualistic insofar as their ontologies apparently have two kinds of entity: the primitive ontology of particles (or what have you) and the quantum state (or wave function). Interpretations of quantum mechanics without a primitive ontology do not have this dualism; therefore, they might seem to have an important advantage over those interpretations which adopt a primitive ontology in addressing this challenge, the extra ontology problem.

This problem can also be (and in a way has been) raised in classical statistical mechanics. Recall that the empirical content of the theories I have been discussing, formally and epistemically-speaking, requires only a set of observables, a physical state in the form of a probability measure, and a dynamics for that state. In quantum mechanics this state is the quantum state and in statistical mechanics it is the statistical state. Whether one opts for a primitive ontology or not, interpreters of statistical mechanics should be able to answer the same challenge: how can one make sense of the statistical state? Indeed, I already showed how one is led directly to this problem in the Boltzmannian and Bohmian interpretations by assuming a primitive ontology from the start. This problem is intimately related to the previously mentioned paradox of deterministic probabilities (Loewer 2001; Winsberg 2008; Lyon 2011)

Let us now proceed a bit further and examine briefly how one might go about solving the extra ontology problem for the evidently dualistic default interpretation of statistical mechanics, the Boltzmannian interpretation. I will simply follow Belot's approach here. Belot says that there are a few possibilities for interpreting the wave function: it is a field (of sorts), a law (of sorts), or a property (of sorts). Let us suppose that a statistical state in statistical mechanics could be interpretable in the same ways and look at them in turn.

If one thinks of the probability distribution $\rho$ as a field, then it must be a field defined on some abstract space (e.g., phase space), making a connection to threedimensional reality difficult. This is also a problem raised against the wave function in quantum mechanics. However, it seems to be even more of a problem in statistical mechanics, since —on the Boltzmannian primitive ontology picture — it is clear that $\rho$ plays no role in the dynamics of the physical particles, unlike in Bohmian mechanics where it "guides" the particles.

If one thinks of the statistical state as instead encoding nomological facts, in analogy to interpretations that take the wave function to do so (Callender 2015), then in the first place it is not so clear what nomological facts it is encoding. In the second, the main complaint against conceiving of the wave function as a nomological entity in quantum mechanics also carries over directly to classical statistical mechanics. That complaint is that wave functions are contingent, since the theory allows that there could be different initial wave functions and since wave functions also evolve in time. This is unlike legitimate laws which hold with physical necessity, such as Hamilton's equation or the Schrödinger equation (Brown and Wallace 2005; 
Belot 2011). As initial statistical states are plainly contingent in the same way and for the same reasons, it does not seem that they can be nomological in nature.

Finally, if one thinks of the statistical state as encoding dispositional properties of a primitive ontology, then one cannot believe this in the Boltzmannian interpretation, for in this case the microscopic physics is deterministic and not dispositional. This is an important difference in comparison to Bohmian quantum mechanics, where the relevant dispositional properties are said to be the velocities of the particles (the wave function is responsible for determining them via the guidance equation). In Boltzmannian statistical mechanics, however, the particles already possess determined velocities by the microscopic dynamics.

Therefore, in all three cases it seems that the extra ontology problem poses a significant problem for the conventional interpretation of statistical mechanics, and this point has not been sufficiently appreciated in the literature. Is it problematic enough to consider alternative interpretations than those that presuppose a primitive ontology?

I happen to believe so. Even if not and these problems can be resolved, it would seem worth at least exploring the interpretive options available. Why not ask, for example, which interpretations are committed to a primitive ontology and which are not? Recall the discussion from the previous section: If one opts for the initial chances approach, then one can (and arguably must) posit a primitive ontology with deterministic dynamics. This is the paradigmatic case which leads to difficulties with the extra ontology problem, as we just saw. If one opts instead for the stochastic dynamics approach, then one can also posit a primitive ontology (although one need not). Unlike the initial chances approach, though, observe that it is possible to characterize the statistical state as encoding dispositional properties, since the microdynamics would not be always deterministic. Perhaps this should be seen as an advantage of this approach. Finally, if one opts for the stochastic observables approach, then one can directly characterize the statistical state as encoding dispositional properties of the macroscopic ontology. It therefore does not face the extra ontology problem (although clearly it does face the challenge of the macro object problem, as does the stochastic dynamics approach without a primitive ontology).

Obviously, there is much more that can and should be said about this cluster of issues. Here is not the place for a lengthy investigation, for my aim has merely been to show the applicability of this typology of interpretations and the analogies between them for linking an important interpretive issue across theories. And so it does: it helpfully reveals how primitive ontology, probability, and determinism become entangled not only in quantum mechanics but in statistical mechanics as well.

\section{The measurement problem}

The second interpretive issue that I wish to consider is the quantum mechanical measurement problem. Just as I claimed that the macro object and extra ontology problems can be posed in statistical mechanics, I claim that a kind of "measurement problem" can be seen to arise in statistical mechanics. I will suggest a reading of the measurement problem in quantum mechanics according to which it is a problem 
about how to interpret the stochasticity of the theory-in which case it should be no surprise that there is an analogous problem in statistical mechanics, for statistical mechanics is a theory which requires an interpretation of stochasticity as well. By suggesting this reading, however, I certainly do not mean to claim that there is nothing more to the quantum measurement problem than a problem of interpreting probabilities in the theory. ${ }^{25}$ Rather, it seems to me that bringing this aspect of the problem to light allows one to be clearer about what distinctively quantum aspects of the problem are actually at issue in quantum mechanics.

Although there are more sophisticated ways one could make the point, I will make use of the simple, well-known presentation of the measurement problem found in Maudlin (1995), specifically what Maudlin calls the "problem of outcomes." He argues that the following three seemingly plausible statements are inconsistent in quantum mechanics:

1. The quantum state of a system is complete; that is, it is sufficient for specifying all of the physical properties of a system.

2. The quantum state always evolves in accord with a deterministic equation, namely the Schrödinger equation.

3. Measurements of observable properties have determinate outcomes; that is, measurements reveal that the system possesses definite properties.

The argument for the inconsistency of these claims is familiar. A generic quantum state is a superposition. Assuming 1 and 2, it follows that a system in a superposition will not in general have determinate outcomes. Assuming 1 and 3, it follows that the system in a superposition cannot have evolved deterministically for it to have determinate properties. Assuming 2 and 3, it follows that the system's state must be supplemented, for something must specify which outcomes obtain in a superposition. According to Maudlin, rejecting 1 leads one to Bohmian mechanics; rejecting 2 leads one to collapse interpretations; rejecting 3 leads one to the many worlds interpretation.

We can contrive a very similar argument in the context of statistical mechanics. I claim that the following three statements are inconsistent:

1. The statistical state of a system is complete; that is, it is sufficient for specifying all of the physical properties of a system.

2. The statistical state always evolves in accord with a deterministic equation, for example the Liouville equation.

3. Measurements of observable properties have determinate outcomes; that is, measurements reveal that the system possesses definite properties.

A generic statistical state is essentially a classical superposition. Assuming 1 and 2 , it follows that a system in such a state cannot have determinate outcomes, for a system in such a superposition does not have determinate properties. Assuming 1

\footnotetext{
${ }^{25}$ Indeed, as a referee points out, the quantum measurement problem is bound up with considerations like "measurement" appearing as a primitive term in the theory, the preferred basis problem, and entanglement, all of which do not have classical analogs.
} 
and 3, it follows that the system cannot have evolved deterministically for it to have determinate outcomes. Assuming 2 and 3, it follows that the system's state must be supplemented by something that specifies the outcomes that actually obtain in the superposition. According to the typology of interpretations of this paper, and in analogy to the quantum mechanical argument, rejecting 1 will lead to some kind of initial chance account, rejecting 2 will lead to some kind of stochastic dynamics account; rejecting 3 will lead to some kind of stochastic observables account.

Presented this way, the manifest issue behind these two measurement problems is how one interprets the stochasticity of a theory of statistical physics. Although there is no doubt that quantum mechanics brings with it distinctive interpretive issues, Maudlin's "problem of outcomes" does not appear to be one of them, for the states of statistical mechanics suffer from the same indeterminateness as quantum states, and the possible solutions to the two problems follow essentially the same lines.

Now, I suppose that some will be little impressed with the revelation of these analogous measurement problems. They will say that this "analogy" is trivial and was already obvious (although one looks in vain for someone making it explicit!) and so too the solution. The very success of statistical mechanics, they might say, demonstrates that the systems it treats are composed of microscopic entities and, indeed, bears out the "atomic hypothesis". There is no question, then, that one should reject the first statement, since systems certainly do possess microscopic states and properties, namely those of atoms and molecules, which can be described in classical mechanics. Unfortunately this popular story is far too sanguine, and in several respects. One is that there are various alternative interpretations available that deserve a fair evaluation and may on balance be preferable to the old story. Two, even if there was no reason to question the ontological presuppositions of classical statistical mechanics in the distant past, surely the acceptance of quantum mechanics as our best microscopic physics should lead us to reconsider what the microscopic entities of classical statistical mechanics really are. It is by no means sure that they are best understood as classical entities. Three, exploring alternative interpretations may suggest novel approaches, techniques, and hypotheses in classical statistical mechanics that would be entirely overlooked by presupposing a resolution to this classical measurement problem.

Finally, it may be of some interest to consider as well how these parallel measurement problems may be solved together, at one stroke, as it were. Although it is possible that one might favor differing solutions for the two theories, it does seem natural to prefer common solutions to both problems, for philosophical reasons or otherwise. Indeed, the Boltzmann-Bohmians see it as an advantage of their position that they have a common primitive ontology in their favored interpretations; it might be seen as an additional virtue that they solve the measurement problems in a consistent way. If it is good wisdom to pursue parallel solutions, then the collapse theorist might look to stochastic dynamics approaches in statistical mechanics, and perhaps doing so might give a useful perspective on the quantum to classical limit. ${ }^{26}$

\footnotetext{
${ }^{26}$ See, e.g., Albert (1994), which suggests underwriting statistical mechanics with the GRW collapse theory.
} 
The same may hold as well for the stochastic observables approaches, perhaps by developing links between Gibbsian statistical mechanics and some kind of Everettian interpretation. $^{27}$

\section{Conclusion}

I have drawn attention to analogies between interpretations of classical statistical mechanics and quantum mechanics centered on an important issue, which I suggest is at the heart of these analogies: the interpretation of stochasticity in the two theories. Rather than discuss probability in all its many traditional interpretations, I focused on realist, ontic interpretations of probability, where probabilities are understood to be related to some kind of fundamental randomness in the world. With this interpretive stance, answering the question of what probability does in statistical theories comes down to locating a source of stochasticity in the theory. I cast statistical mechanics and quantum mechanics in a common abstract form, starting from the epistemically significant empirical content of the theories, the statistics of observables. Stochasticity, I argued, could be associated with three aspects of the theories: its initial conditions, its dynamics, or its observable properties. As it turns out, the usual "realist" interpretations of the two theories can be seen to line up closely with these choices. The most well-known analogy, that between Boltzmannian statistical mechanics and Bohmian quantum mechanics, takes the first option; stochastic dynamics approaches in statistical mechanics and collapse interpretations in quantum mechanics take the second; the third option is unusual in that it has been for the most part overlooked in interpretational work, although I suggested that Gibbsian statistical mechanics and many worlds quantum mechanics might be understood to take this option, were one to give their probabilities some sort of ontic interpretation.

To show that this way of thinking about the two theories is fertile, I discussed its consequences for two major interpretive issues in quantum mechanics: primitive ontology and the measurement problem. I argued that debates about these issues could be reprised in the context of statistical mechanics. Moving the debate about primitive ontology into statistical mechanics puts to question, for example, the nature of a statistical state: is it an object, a law, a property? I also argued that at least one popular rendering of the quantum measurement problem translates directly into statistical mechanics, which I took to reveal that to some extent what is called the quantum measurement problem involves a problem of making sense of its probabilities. I showed that analogous interpretations of the two theories solve the two measurement problem in much the same way, which draws further attention to the generally overlooked intertheoretical relation between quantum mechanics and statistical mechanics (instead of that between quantum mechanics and classical mechanics).

\footnotetext{
${ }^{27}$ Something of the sort seems to be akin to Wallace's interpretive stance in recent papers on quantum and statistical mechanics, for example Wallace (2014, 2016a, 2018).
} 
Acknowledgements Thanks to Bryan Roberts, Miklós Rédei, and John Dougherty for valuable discussions related to this paper. Some of this material was presented at a meeting of the Sigma Club in London and at the Sixth European Philosophy of Science Association Biennial Meeting in Exeter; I thank the audiences at these talks, as well as this journal's referees for their comments and suggestions.

Funding Information Open access funding provided by Stockholm University.

Open Access This article is distributed under the terms of the Creative Commons Attribution 4.0 International License (http://creativecommons.org/licenses/by/4.0/), which permits unrestricted use, distribution, and reproduction in any medium, provided you give appropriate credit to the original author(s) and the source, provide a link to the Creative Commons license, and indicate if changes were made.

\section{References}

Albert, D. (1994). The foundations of quantum mechanics and the approach to thermodynamic equilibrium. Erkenntnis, 41, 191-206.

Albert, D. (2000). Time and chance. Cambridge: Harvard University Press.

Albert, D. (2013). Wave function realism. In A. Ney, \& D. Albert (Eds.) The wave function (pp. 52-57). New York: Oxford University Press.

Allori, V., Goldstein, S., Tumulka, R., Zanghì, N. (2014). Predictions and primitive ontology in quantum foundations: a study of examples. The British Journal for the Philosophy of Science, 65, 323352.

Allori, V., \& Zanghì, N. (2004). What is Bohmian mechanics. International Journal of Theoretical Physics, $43,1743-1755$.

Ballentine, L. (1970). The statistical interpretation of quantum mechanics. Reviews of Modern Physics, $42,358-381$.

Bell, J. (1982). On the impossible pilot wave. Foundations of Physics, 12, 989-999.

Bell, J. (2004). The theory of local beables. In Speakable and unspeakable in quantum mechanics (pp. 5262). Cambridge: Cambridge University Press.

Belot, G. (2011). Quantum states for primitive ontologist. European Journal for Philosophy of Science, 2, 67-83.

Brown, H., \& Wallace, D. (2005). Solving the measurement problem: De Broglie-Bohm loses out to Everett. Foundations of Physics, 35, 517-540.

Callender, C. (2007). The emergence and interpretation of probability in Bohmian mechanics. Studies in History and Philosophy of Modern Physics, 38, 351-370.

Callender, C. (2015). One world, one beable. Synthese, 192, 3153-3177.

Callender, C., \& Weingard, R. (1997). Trouble in paradise? Problems for Bohm's theory. The Monist, 80, 24-43.

Daumer, M., Dürr, D., Goldstein, S., Zanghì, N. (1996). Naive realism about operators. Erkenntnis, 45, 379-397.

Demarest, H. (2016). The universe had one chance. Philosophy of Science, 83, 248-264.

Dürr, D. (2001). Bohmian mechanics. 2001. In J. Bricmont, D. Dürr, M. Galavotti, G. Ghirardi, F. Petruccione, N. Zanghì (Eds.) Chance in physics (pp. 115-131). Berlin: Springer.

Dürr, D., Goldstein, S., Zanghì, N. (1992). Quantum equilibrium and the origin of absolute uncertainty. Journal of Statistical Physics, 67, 843-907.

Dürr, D., Goldstein, S., Zanghì, N. (1995). Quantum physics without quantum philosophy. Studies in History and Philosophy of Modern Physics, 26, 137-149.

Dürr, D., \& Teufel, S. (2009). Bohmian mechanics. Berlin: Springer.

Egg, M., \& Esfeld, M. (2015). Primitive ontology and quantum state in the GRW matter density theory. Synthese, 192, 3229-3245.

Esfeld, M. (2014). The primitive ontology of quantum physics: guidelines for an assessment of the proposals. Studies in History and Philosophy of Modern Physics, 47, 99-106.

Esfeld, M., \& Gisin, N. (2014). The GRW flash theory: a relativistic quantum ontology of matter in spacetime? Philosophy of Science, 81, 248-264. 
Esfeld, M., Hubert, M., Lazarovici, D., Dürr, D. (2014). The ontology of Bohmian mechanics. The British Journal for the Philosophy of Science, 65, 773-796.

Frigg, R. (2009). Typicality and the approach to equilibrium in Boltzmannian statistical mechanics. Philosophy of Science, 76, 997-1008.

Frigg, R., \& Hoefer, C. (2015). The best Humean system for statistical mechanics. Erkenntnis, 80, 551574.

Frigg, R., \& Werndl, C. (2018). Forthcoming. Can somebody please say what Gibbsian statistical mechanics says? The British Journal for the Philosophy of Science.

Ghirardi, G. (2016). Collapse theories. In Zalta, E. (Ed.) The Stanford encyclopedia of philosophy. Spring 2016 edition. http://plato.stanford.edu/archives/spr2016/entries/qm-collapse/.

Goldstein, S. (2001). Boltzmann's approach to statistical mechanics. In J. Bricmont, D. Dürr, M. Galavotti, G. Ghirardi, F. Petruccione, N. Zanghì (Eds.) Chance in physics (pp. 39-54). Berlin: Springer.

Goldstein, S. (2012). Typicality and notions of probability in physics. In Y. Ben-Menahem, \& M. Hemmo (Eds.) Probability in physics (pp. 59-71). Berlin: Springer.

Goldstein, S. (2017). Bohmian mechanics. In E. Zalta (Ed.) The Stanford encyclopedia of philosophy. Summer 2017 edition. https://seop.illc.uva.nl/entries/qm-bohm/.

Greaves, H. (2007). Probability in the Everett interpretation. Philosophy Compass, 2, 109-128.

Griffiths, R. (2019). The consistent histories approach to quantum mechanics. In E. Zalta (Ed.) The Stanford encyclopedia of philosophy. Summer 2019 edition. https://plato.stanford.edu/archives/sum2019/ entries/qm-consistent-histories/.

Landsman, K. (2017). Foundations of quantum theory. Cham: Springer.

Lavis, D. (1977). The role of statistical mechanics in classical physics. The British Journal for the Philosophy of Science, 28, 255-279.

Lewis, D. (1973). Counterfactuals. Cambridge: Harvard University Press.

Lewis, D. (1981). A subjectivist's guide to objective chance. In W. Harper, R. Stalnaker, G. Pearce (Eds.) IFS (pp. 267-297). Dordrecht: D. Reidel Publishing Company.

Lewis, D. (1983). New work for a theory of universals. Australasian Journal of Philosophy, 61, 343377.

Loewer, B. (1996). Humean supervenience. Philosophical Topics, 24, 101-126.

Loewer, B. (2001). Determinism and chance. Studies in History and Philosophy of Modern Physics, 32, $609-620$.

Loewer, B. (2004). David Lewis's Humean theory of objective chance. Philosophy of Science, 71, 11151125 .

Luczak, J. (2016). On how to approach the approach to equilibrium. Philosophy of Science, 83, 393-411.

Lyon, A. (2011). Deterministic probability: neither chance nor credence. Synthese, 182, 413-432.

Maudlin, T. (1995). Three measurement problems. Topoi, 14, 7-15.

Maudlin, T. (2007a). Completeness, supervenience and ontology. Journal of Physics A, 40, 3151-3171.

Maudlin, T. (2007b). What could be objective about probabilities? Studies in History and Philosophy of Modern Physics, 38, 275-291.

Maudlin, T. (2011). Three roads to objective probability. In C. Beisbart, \& S. Hartmann (Eds.) Probabilities in physics (pp. 293-319). Oxford: Oxford University Press.

McCoy, C. (2018a). No chances in a deterministic world. PhilSci archive. http://philsci-archive.pitt.edu/ 13166/.

McCoy, C. (2018b). Forthcoming. An alternative interpretation of statistical mechanics. Erkenntnis.

Ney, A., \& Albert, D. (Eds.) (2013). The wave function. Oxford: Oxford University Press.

Ney, A., \& Phillips, K. (2013). Does an adequate physical theory demand a primitive ontology? Philosophy of Science, 80, 454-474.

North, J. (2013). The structure of the quantum world. In A. Ney, \& D. Albert (Eds.) The wave function (pp. 184-202). New York: Oxford University Press.

Rédei, M., \& Summers, S. (2007). Quantum probability theory. Studies in History and Philosophy of Modern Physics, 38, 390-417.

Saunders, S. (2010). Chance in the Everett interpretation. In S. Saunders, J. Barrett, A. Kent, D. Wallace (Eds.) Many worlds? Everett, quantum theory, \& reality (pp. 181-205). Oxford: Oxford University Press.

Sebens, C., \& Carroll, S. (2016). Self-locating uncertainty and the origin of probability in Everettian quantum mechanics. The British Journal for the Philosophy of Science, 69, 25-74.

Sklar, L. (1993). Physics and chance. Cambridge: Cambridge University Press. 
Souriau, J.-M. (1997). Structure of dynamical systems. New York: Springer.

Uffink, J. (2007). Compendium of the foundations of classical statistical physics. In J. Buttefield, \& J. Earman (Eds.) Philosophy of physics (pp. 923-1074). Amsterdam: Elsevier.

Wallace, D. (2012). The Emergent multiverse. Oxford: Oxford University Press.

Wallace, D. (2014). Probability in physics: stochastic, statistical, quantum. In A. Wilson (Ed.) Chance and temporal asymmetry (pp. 194-220). Oxford: Oxford University Press.

Wallace, D. (2015). The quantitative content of statistical mechanics. Studies in History and Philosophy of Modern Physics, 52, 285-293.

Wallace, D. (2018). The necessity of Gibbsian statistical mechanics. PhilSci archive. http://philsci-archive. pitt.edu/15290/.

Wallace, D. (2016a). Forthcoming a. Probability and irreversability in modern statistical mechanics: classical and quantum. In D. Bedingham, O. Maroney, C. Timpson (Eds.) Quantum foundations of statistical mechanics. Oxford: Oxford University Press.

Wallace, D. (2016b). Forthcoming b. What is orthodox quantum mechanics? In Proceedings of the XII international ontology congress.

Wallace, D., \& Timpson, C. (2010). Quantum mechanics on spacetime i: spacetime state realism. The British Journal for the Philosophy of Science, 61, 697-727.

Werndl, C. (2009). Are deterministic descriptions and indeterministic descriptions observationally equivalent? Studies in History and Philosophy of Modern Physics, 40, 232-242.

Werndl, C. (2011). On the observational equivalence of continuous-time deterministic and indeterministic descriptions. European Journal for Philosophy of Science, 1, 193-225.

Winsberg, E. (2008). Laws and chances in statistical mechanics. Studies in History and Philosophy of Modern Physics, 39, 872-888.

Publisher's note Springer Nature remains neutral with regard to jurisdictional claims in published maps and institutional affiliations. 